\title{
Rapid modulation of the silent information regulator 1 (SIRT1) by melatonin after hypoxia-ischemia in the neonatal rat brain
}

Silvia Carloni $^{1 *} \mid$ Giulia Riparini $^{1}$ | Giuseppe Buonocore ${ }^{2}$ | Walter Balduini $^{1}$

${ }^{1}$ Department of Biomolecular Sciences, University of Urbino "Carlo Bo", Via S. Chiara 27, 61029 Urbino, Italy.

${ }^{2}$ Department of Molecular and Developmental Medicine, Policlinico Le Scotte, University of Siena, Siena, Italy.

*Silvia Carloni (Corresponding author)

Department of Biomolecular Sciences, University of Urbino “Carlo Bo" Via S. Chiara 27, 61029, Urbino (PU), Italy.

Tel. +39-0722-303528; Fax: +39-0722-303521

e-mail: silvia.carloni@uniurb.it

Running title: SIRT1 and melatonin in neonatal brain ischemia

Keywords: SIRT1, melatonin, hypoxia-ischemia, neuroprotection 


\begin{abstract}
Increasing evidence indicates that melatonin possesses protective effects toward different kinds of damage in various organs, including the brain. In a neonatal model of hypoxia-ischemia (HI), melatonin was neuroprotective and preserved the expression of the Silent Information Regulator 1 (SIRT1) $24 \mathrm{~h}$ after the insult. This study aimed to gain more insight into the role of SIRT1 in the protective effect of melatonin after HI by studying the early (1h) modulation of SIRT1 and its downstream targets, and the consequences on necrosis, apoptosis, autophagy and glial cell activation. We found that melatonin administered 5 min after the ischemic insult significantly reduced necrotic cell death assessed $1 \mathrm{~h}$ after its administration. In parallel, we found a reduced activation of the early phases of intrinsic apoptosis, detected by reduced Bax translocation to the mitochondria and preservation of the mitochondrial expression of cytochrome $\mathrm{C}$, indicating a reduced outer mitochondrial membrane permeabilization in the melatonin-treated ischemic animals. These effects were concomitant to increased expression and activity of SIRT1, reduced expression and acetylation of p53, and increased autophagy activation. Melatonin also reduced HI-induced glial cells activation. SIRT1 was expressed in neurons after HI and melatonin but not in reactive glial cells expressing GFAP. Co-localization between SIRT1 and GFAP was found in some cells in control conditions. In summary, our results gain more insight into the connection between SIRT1 and melatonin in neuroprotection. We suggest that increased SIRT1 activity induced by melatonin may likely contribute to differentiate neuronal progenitor cells during the neurodegenerative process.
\end{abstract}




\section{1 | INTRODUCTION}

Melatonin (N-acetyl-5-methoxytryptamine) is a versatile and ubiquitous molecule with pronounced protective effects in several models of perinatal brain damage. ${ }^{1}$ Melatonin has also shown promising therapeutic activity in preterm-delivered newborns and in the treatment of neonates and preterm newborns affected by disorders such as sepsis, asphyxia, respiratory distress and surgical complications..$^{2-3}$ The therapeutic effects of melatonin have been ascribed to its potent antioxidant activity $^{4-5}$ and its pleiotropic effects affecting epigenetic regulation, ${ }^{6}$ energy metabolism ${ }^{7}$ and immune function. ${ }^{8}$ Recently, melatonin was found to strongly affect the modulation of SIRT1, a nicotinamide adenine dinucleotide (NAD+)-dependent deacetylase, in different models of perinatal brain injury. ${ }^{9-10}$ SIRT1 belongs to a family of proteins with homologies to the Silence Information Regulator 2 (SIR2) gene of the yeast S. Cerevisiae, ${ }^{11}$ known as sirtuins. In mammals, seven sirtuins have been identified (SIRT1-7). SIRT1, SIRT6, and SIRT7 are mainly found in the nucleus, SIRT3, SIRT4, SIRT5 in the mitochondrion, and SIRT2 in the cytoplasm. However, sirtuins can also translocate to or shuttle among subcellular compartments. ${ }^{12}$ SIRT1 is the most studied sirtuin. Deacetylation of lysine by SIRT1 is coupled to the cleavage and content of NAD+, a key intermediate of cellular energy metabolism, and is inhibited by the reaction product nicotinamide. This makes SIRT1 activity effectively influenced by the metabolic and redox conditions of the cell. Two groups of acetylated proteins are its main targets, i.e. histone and non-histone proteins. ${ }^{13}$ Histone protein regulation by SIRT1 affects chromatin access for gene transcription, whereas non-histone substrates are generally molecules or enzymes that control signal transduction, metabolism, and gene transcription. ${ }^{14}$ SIRT1 regulates cellular metabolism by modulating the nutrient sensing pathways controlled by AMPdependent kinase (AMPK) ${ }^{15}$ and the mammalian target of rapamycin (mTOR), ${ }^{16}$ affecting, therefore, the autophagy process and the UPR response. ${ }^{17}$ The transcription factor and tumor suppressor p53, 
FOXO proteins and Nf-kB are also targets of SIRT1. ${ }^{18-19}$ Deacetylation of p53 by SIRT1 provides a quick acting mechanism to stop its function once transcriptional activation of target genes is no longer needed and it inhibits apoptosis. ${ }^{18,20}$ A role of SIRT1 in differentiation of neuronal progenitor cells $(\mathrm{NPCs})^{21-22}$ and promotion of oligodendrocyte progenitor cell proliferation has also been reported. ${ }^{23}$ By influencing chromatin structure, gene transcription, metabolism, inflammation, apoptosis and NPCs differentiation, SIRT1 may play a key role in neurodegeneration and neuroprotection and may also modulate the scar formation associated with the neurodegenerative process. ${ }^{24}$

We recently found that after neonatal hypoxia-ischemia (HI) in pup rats, SIRT1 expression was significantly reduced in cells that activate the Unfolded Protein Response (UPR), ${ }^{9}$ a cellular response aimed to overcome endoplasmic reticulum (ER) stress. We also found that melatonin completely prevents the HI-induced SIRT1 depletion and UPR activation, indicating a possible role of SIRT1 in the neuroprotective effect of melatonin. However, these effects were assessed 24 hours after HI, when the complex mechanisms underlying the neurodegenerative process are in a late phase. Here, we studied the early effects of ischemia and melatonin on the modulation of SIRT1 and its downstream targets along with the subsequent consequences on necrosis, apoptosis, autophagy and glial cell activation. 


\section{2 | METHODS}

\section{1 | Cerebral hypoxia-ischemia (HI).}

All surgical and experimental procedures were carried out in accordance with the Italian regulation for the care and use of laboratory animals (according to the EU Directive 2010/63/EU), and were approved by the Animal Care Committee of the University of Urbino "Carlo Bo". Pregnant SpragueDawley rats were housed in individual cages and the day of delivery was considered day 0 . Neonate rats from different litters were randomized, normalized to ten pups per litter and kept in regular light/dark cycle (lights on 8 am-8 pm). On postnatal day 7 , after anesthesia with $5 \%$ isoflurane in $\mathrm{N}_{2} \mathrm{O} / \mathrm{O}_{2}(70 / 30 \%)$ mixture, pup rats underwent unilateral ligation of the right common carotid artery via a midline neck incision. After artery ligation, the wound was sutured and the animals allowed to recover for $3 \mathrm{~h}$ under a heating lamp. Pups were then placed in an airtight jar and exposed for $2.5 \mathrm{~h}$ to a humidified nitrogen-oxygen mixture ( $92 \%$ and $8 \%$, respectively) delivered at 5-6 L/min (HI). The jar was partially submerged in a $37^{\circ} \mathrm{C}$ water bath to maintain a constant thermal environment.

\section{2 | Drugs administration.}

Melatonin (Sigma-Aldrich, Milan, Italy, M5250) was dissolved in dimethyl sulfoxide (DMSO; Sigma-Aldrich, Milan, Italy, D5879) and diluted in normal saline solution to a final concentration of 5\% DMSO (vehicle). The melatonin solution was injected intraperitoneally to pup rats 5 min after HI at the dose of $15 \mathrm{mg} / \mathrm{kg}$. Control animals received the same volume of vehicle. Propidium iodide (PI; $0.5 \mu \mathrm{L}$ of a $1 \mathrm{mg} / \mathrm{mL}$ solution in distilled water; Sigma-Aldrich, Milan, Italy, P4170) was injected into the right lateral ventricle. Twenty minutes after PI injection, animals were sacrificed and perfusion-fixed with $4 \%$ paraformaldehyde in $0.1 \mathrm{~mol} / \mathrm{L} \mathrm{PBS}{ }^{25}$ 


\section{3 | Western blot analysis.}

Pups were anesthetized and euthanized by decapitation. Brains were rapidly removed and the cytosolic, mitochondrial and nuclear fractions prepared from cerebral cortices according to Nijboer et al. ${ }^{26}$ Samples were stored at $-80^{\circ} \mathrm{C}$ until used. After mixing with sodium dodecyl sulfate gel-loading buffer and heating $4 \mathrm{~min}$ at $95^{\circ} \mathrm{C}$, samples (20-50 $\mu$ g protein) were electrophoresed onto sodium dodecyl sulfate-polyacrylamide gel and proteins transferred to a PVDF membrane. ColorBurst ${ }^{\mathrm{TM}}$ electrophoresis marker ( $3 \mu \mathrm{L} /$ gel, Sigma-Aldrich, Milan, Italy, C1992) was used for qualitative molecular mass determinations and for visual confirmation of blot transfer efficiency. Blots were then blocked with non-fat dry milk in TBS-T (10 mM Tris, $150 \mathrm{mM} \mathrm{NaCl,} \mathrm{pH} \mathrm{7.6,} \mathrm{plus} 0.1 \%$ Tween-20) and probed with the following primary antibodies: anti-SIRT1 (1:1000, polyclonal; Santa Cruz Biotechnology, Dallas, TX, USA, sc-15404), anti-cytochrome c (1:1000, monoclonal; Santa Cruz Biotechnology, Dallas, TX, USA, sc-7159), anti-Bax (1:500, monoclonal; Santa Cruz Biotechnology, Dallas, TX, USA, sc-7480), anti-cleaved caspase-3 (1:1000, polyclonal; Cell Signaling Technology, Danvers, MA, USA, \#9661), anti-p53 (1:2000, monoclonal; Santa Cruz Biotechnology, Dallas, TX, USA, sc-126), anti-acetyl-p53 (1:500, polyclonal; Cell Signaling Technology, Danvers, MA, USA, \#2525), anti-LC3 (1:1000, polyclonal; Cell Signaling Technology, Danvers, MA, USA, \#2775), antip62 (1 $\mu \mathrm{g} / \mathrm{mL}$, polyclonal; Sigma-Aldrich, P0067) and anti-GFAP (1:2000, monoclonal; Boehringer Mannheim GmbH, Germany, 813 369). A monoclonal antibody against $\beta$-actin (1:4000, Santa Cruz Biotechnology, Dallas, TX, USA, sc-8432) or against Hsp60 (1:1000, Santa Cruz Biotechnology, Dallas, TX, USA, sc-13115) and a polyclonal antibody against Lamin B (1:1000, Santa Cruz Biotechnology, Dallas, TX, USA, sc-6216) were used as controls for protein gel loading. Data were normalized to $\beta$-actin or Hsp60 or lamin B, and expressed as \% of control.

\section{4 | Immunohistochemistry.}


One hour after HI, pups were deeply anesthetized with $5 \%$ isoflurane in $\mathrm{N}_{2} \mathrm{O} / \mathrm{O}_{2}(70 / 30 \%)$ mixture and perfusion-fixed with $4 \%$ paraformaldehyde in $0.1 \mathrm{~mol} / \mathrm{L}$ PBS. Brains were rapidly removed on ice and processed for antigen retrieval by immersing overnight in $10 \mathrm{mmol} / \mathrm{L}$ sodium citrate buffer (pH 6.0, $4^{\circ} \mathrm{C}$ ) and boiling in the same buffer for $3 \mathrm{~min}$. After boiling, brains were cryoprotected with $30 \%$ sucrose/PBS $\left(72 \mathrm{~h}, 4^{\circ} \mathrm{C}\right)$. Brain sections (thickness $12 \mu \mathrm{m}$ ) were incubated with $1.5 \%$ normal blocking serum for $1 \mathrm{~h}$ at room temperature, and then overnight at $4^{\circ} \mathrm{C}$ with anti-SIRT1 $(1: 50$, polyclonal; Santa Cruz Biotechnology, Dallas, TX, USA, sc-15404), anti-GFAP (1:300, monoclonal; Boehringer Mannheim GmbH, Germany, 813 369) or anti-neuron-specific nuclear protein (NeuN, 1:500; monoclonal; Millipore, Billerica, MA, USA, MAB377). Fluorescein IsoThiocyanateconjugated mouse anti-rabbit IgG (1:200; Santa Cruz Biotechnology, Dallas, TX, USA, sc-2359) and Texas Red anti-mouse IgG (1:200; Santa Cruz Biotechnology, Dallas, TX, USA, sc-3842) were used to demonstrate immunoreactivity of SIRT-1 as green fluorescence and of NeuN or GFAP as red fluorescence, respectively. In some experiments, GFAP immunoreactivity was detected with a biotinylated goat anti-mouse antibody (1:200) and visualized using avidin-biotin peroxidase solution (Elite ABC kit, Vectastain Vector, Vector Laboratories, Burlingame, CA, USA, PK6101). Peroxidase activity was evidenced by $0.05 \% 3,3$ '-diaminobenzidine tetrahydrochloride (DAB) and $0.03 \% \mathrm{H}_{2} \mathrm{O}_{2}$ at the appropriate stage. The specificity of the reactions was evaluated in some slices by omitting the primary antibody from the incubation medium.

\section{5 | Cell counting.}

Cell counting after PI injection was conducted in the cerebral cortex and in the CA1 and CA2/CA3 areas of the hippocampus on 20X microscopic images using a BX-51 Olympus microscope. Positive cells were counted in three separate fields of each area in slices cut at the level A 3750 of the Konig and Klippel stereotaxic atlas. ${ }^{25}$ Five animals per group were analyzed. 


\section{6 | Data analysis.}

The relative intensity of the bands detected by Western blot was analyzed using the Image J 1.45 software. Statistical analyses were performed by One-way ANOVA using Prism Computer program (GraphPad Software Inc.). The Newman-Keuls multiple comparison test was used to determine differences between single treatment groups. $\mathrm{P} \leq 0.05$ was considered significant. 


\section{3 | RESULTS}

\section{1 | Melatonin reduces HI-induced necrotic and apoptotic cell death}

To assess the early effects of melatonin administered $5 \mathrm{~min}$ after the neonatal hypoxic-ischemic insult, we assessed necrotic and apoptotic outcomes in animals sacrificed 1 hour after HI. Necrosis was assessed by PI injection, a fluorescent dye that we previously used to follow the time-course of necrotic cell death and apoptosis-to-necrosis transition in this model of HI. ${ }^{25,27}$ PI was injected icv 20 min before sacrificing the animals for biochemical analyses. As shown in Fig. 1, PI-positive cells were observed in the CA1 area of the hippocampus. A lower number of PI-positive cells was detected in the cerebral cortex. In this brain area, PI-positive cells were detected mainly in the deep layers (Fig. 1A). In melatonin-treated ischemic animals, the number of PI-positive cells was lower both in the hippocampus and in the cortex (Fig. 1A). The reduced amount of PI-positive cells after melatonin was confirmed by cell counting (Fig 1B), indicating that melatonin reduces necrotic cell death after the insult.

Apoptotic cell death was studied by assessing cleaved caspase-3 expression. However, $1 \mathrm{~h}$ after HI, cleaved caspase-3 was still undetectable (data not shown). Therefore, we addressed our experiments to the evaluation of Bax translocation to the mitochondria and the mitochondrial release of cytochrome $\mathrm{C}$, which are affected by activation of the intrinsic apoptotic pathway and precede caspase-3 activation. As reported in Figure 2, there was an increased Bax translocation to the mitochondria (Fig. 2A and B) and a significant release of cytochrome $\mathrm{C}$ into the cytosol (Fig. 2C and 2D) in the lesioned side of the brain of ischemic animals. Treatment with melatonin significantly reduced Bax translocation to the mitochondria (Fig. 2A and B) and preserved the mitochondrial expression of cytochrome C (Fig. 2C and 2D), indicating a reduced outer membrane permeabilization in melatonin-treated ischemic animals. The anti-apoptotic effect of melatonin was still observed $6 \mathrm{~h}$ 
after HI. At this time, the expression of cleaved caspase-3 was already detectable in the ischemic cortex and was found to be significantly decreased in animals treated with melatonin (Fig 2E).

\subsection{Melatonin preserves SIRT1 expression}

We previously found that SIRT1 is strongly modulated $24 \mathrm{~h}$ after HI. ${ }^{9}$ To assess if SIRT1 modulation represents an early effect of $\mathrm{HI}$ and melatonin administration, we studied this modulation $1 \mathrm{~h}$ after $\mathrm{HI}$. Figure 3 shows immunofluorescence experiments of SIRT1 in the lesioned side of the cerebral cortex and the CA1 area of the hippocampus. Few SIRT1-positive cells were detectable in the lesioned cortex of ischemic animals (Fig. 3A, panel a). SIRT1-positive cells, on the other hand, were appreciably in higher amount in the lesioned side of melatonin-treated ischemic animals (Fig. 3A, panel b). A comparable situation was found in the CA1 area of the hippocampus (Fig. 3A). In this region, the effects of HI (Fig. 3A, panel c) and melatonin (Fig. 3A, panel d) on SIRT1 expression were more pronounced. The modulation of SIRT1 was also confirmed by Western blot experiments performed in cortical tissues. No changes in SIRT1 expression were found in vehicle-treated and melatonin-treated control animals nor in the contralateral cortex of vehicle-treated and melatonintreated ischemic animals (Fig. 3B). Conversely, SIRT1 expression was significantly reduced in the lesioned cortex of ischemic animals (51.9 $\pm 8.1 \%$ reduction versus control group, $\mathrm{p} \leq 0.001$; Fig. 3B). SIRT1 expression was preserved in melatonin-treated ischemic animals (10.6 $\pm 2.4 \%$ reduction versus control group, $\mathrm{p} \geq 0.05$; Fig. 3B). Since SIRT1, initially described as a nuclear protein, ${ }^{28}$ may also shuttle to the cytoplasm, we assessed its modulation in both the nuclear and cytosolic subcellular fractions. As shown in Fig 3, SIRT1 was expressed both in the cytosolic and in the nuclear fractions (Fig. 3C and D), although integrated optical density (OD) measurement of samples run in the same gel showed that the highest amount of the protein was observed in the nucleus (Figure 3C and D). In control conditions, melatonin did not affect SIRT1 expression in both subcellular fractions (Fig. 3C 
and D). After HI, however, the expression of the protein was significantly decreased in both subcellular fractions. Melatonin increased SIRT1 expression in both fractions (Fig. 3C and D).

\section{3 | Melatonin modulates p53}

To determine if the expression of SIRT1 was related to its activation, we assessed the acetylation status of the tumor suppressor p53, a well-known SIRT1 downstream target. ${ }^{18,29}$ As shown in Figure 4, HI significantly increased the cytosolic and nuclear expression of p53 (Fig. 4A and B). The same trend was detected for its acetylated form (Fig. 4C and D). After melatonin, p53 expression and acetylation did not differ from controls in both the cytosol and the nucleus (Fig 4).

\section{4 | Melatonin affects autophagy activation}

SIRT1 also plays a role in autophagy activation. ${ }^{30}$ Thus, we tested if the increased SIRT1 expression is associated with the modulation of autophagy. As shown in Fig 5, HI significantly increased LC3 II and decreased p62 expression (Fig. 5A and B, respectively), indicating autophagy activation. Melatonin significantly increased the HI-induced LC3 II expression and reduced expression of p62 (Fig 5A and B, respectively). These effects are indicative of autophagy over-activation after melatonin in ischemic conditions.

\subsection{Melatonin reduces glial cell activation}

We next investigated the effect of melatonin on glial cell activation. As shown in Figure 6, HI increased the glial fibrillary acidic protein (GFAP) expression, indicating a rapid glial cells activation after HI. Melatonin significantly reduced the HI-induced GFAP expression (Fig. 6A and B).

\section{6 | SIRT1 increases in neurons but not in reactive glia cells after HI}


To assess if both neurons and glial cells express SIRT1 after HI, we performed double-staining immunohistochemical experiments. As shown in Fig. 7A, reactive glial cells expressing GFAP did not express SIRT1 (Fig. 7A, panel f), although we found a co-localization between SIRT1 and GFAP in control conditions (Fig. 7A, panel c). Some double-positive cells were again detectable in the ischemic tissue of melatonin treated animals (Fig. 7A, panel i). In contrast, SIRT1 co-localized with the neuronal-specific nuclear marker NeuN in control and HI conditions (Fig. 7B, panels n and q), as well as in ischemic tissue treated with melatonin (Fig. 7B, panel t). 


\section{4 | DISCUSSION}

We report here that SIRT1 and its downstream pathways are rapidly modulated after neonatal HI. This rapid modulation was concomitant with autophagy activation and found only in the ischemic side of the brain. Pharmacological doses of melatonin administered five minutes after the end of the hypoxic ischemic insult rapidly and completely reversed the effect of HI on SIRT1 and potentiated autophagy. The effects of melatonin were associated with reduction of necrotic cell death and activation of the intrinsic pathway of apoptosis. In addition, melatonin also reduced glial cell activation. These data expand our previous findings showing that melatonin reduced the unfolded protein response induced by neonatal HI and preserved SIRT1 expression 24 hours after the insult. ${ }^{9}$ A similar effect was also found in a perinatal model of brain inflammation induced by LPS. ${ }^{10}$

\section{1 | SIRT1 in neurodegeneration and its modulation by melatonin}

Protein acetylation is emerging as an evolutionarily conserved regulatory mechanism involved in coordinating a variety of metabolic pathways in response to different conditions, including glycolysis, fatty acid synthesis, gluconeogenesis, cell cycle, DNA repair, cell survival and differentiation, mitochondrial biogenesis and autophagy. ${ }^{22,31-33}$ This is achieved by epigenetic modulation of histone and nonhistone proteins in the nucleus, to regulate gene expression, and by modulation of specific protein substrates within the mitochondria and the cytoplasm. ${ }^{14}$ Here we report that in 7 day-old rats SIRT1 is predominantly localized in the nucleus, although it is also present in significant amounts in the cytosol. We also show that SIRT1 expression is rapidly modulated in both compartments, and by both HI and melatonin. The distribution and modulation by HI and melatonin supports a role of SIRT1 in controlling cell activity and neurodegeneration in both cellular compartments. The effect of HI and melatonin on SIRT1 was found in the ischemic side of the brain but not in the contralateral side that, 
in this model of neonatal HI, is hypoxic but not ischemic. This finding indicates that hypoxia, which by itself is unable to cause injury in our experimental model, does not affect the expression and activity of the sirtuin, thus supporting the relationship between reduction of SIRT1 and neurodegeneration. In our study, we did not assess the effect of SIRT1 inhibitors on injury outcome. However, there is already clear evidence in literature demonstrating that activation or inhibition of SIRT1 modulates cerebral outcomes after ischemia. For example, SIRT1 ${ }^{-/}$mice display larger infarcts compared to the wild-type, and treatment with a SIRT1 activator (Activator 3) reduces infarct volume, whereas treatment with a sirtuin inhibitor (sirtinol) increases the infarct volume to vehicle levels after pMCAo. ${ }^{34}$ Interestingly, Activator 3 also inactivated $\mathrm{p} 53,{ }^{34}$ whose shuttling and activity are also regulated by reversible acetylation. ${ }^{35}$ In agreement with these findings, we show here that SIRT1 inactivation was concomitant with increased expression and acetylation of p53, which was reported to control values through melatonin administration.

Sirtuins can directly influence autophagy via deacetylation of key components of the autophagy induction network (Atg proteins) or through the activation of FoxO transcription factor family members, the AMP-dependent kinase or the mammalian target of rapamycin. ${ }^{17}$ In the heart, increased SIRT1 expression and activation by resveratrol can protect cardiomyocytes via autophagy induction. ${ }^{36}$ Autophagy is increased by several treatments that protect the brain after $\mathrm{HI}^{27,37-38}$ and is restored by melatonin after brain inflammation. ${ }^{10}$ In addition, melatonin also reduced HI-induced ER stress, a cellular response strictly connected to autophagy and associated to reduction of SIRT1 expression. ${ }^{9}$ The finding that SIRT1 and autophagy are concomitantly upregulated by different kinds of insults, ${ }^{39-40}$ indicates a connection between SIRT1 upregulation and autophagy, and their concomitant modulation can play an important role in the complex and dynamic signaling network involved in neuroprotection. 


\subsection{Implication of SIRT1 in neuronal survival and glial cell activation and the}

\section{effect of melatonin}

Here, we also found a clear activation of glial cells as early as $1 \mathrm{~h}$ after HI. Pharmacological doses of melatonin significantly reduced the effect of $\mathrm{HI}$ on glial cell activation and protected from injury. Interestingly, we observed that reactive astrocytes did not express SIRT1, as shown by immunohistochemical double-staining experiments, although co-localization between SIRT1 and GFAP was found in some cells in control conditions and in the ischemic tissue of melatonin treated animals. SIRT1, conversely, co-localized with the neuronal-specific nuclear marker NeuN both in control and $\mathrm{HI}$ conditions, as well as in the ischemic side of animals treated with melatonin. These results are in keeping with those previously reported by Hernàndez-Jiménez after permanent focal ischemia in adult mice; Hernàndez-Jiménez and colleagues also found that SIRT1 was present in neurons but not in GFAP-positive astrocytes. ${ }^{34}$

Rapidly-emerging evidence suggests that in the brain, SIRT1 could be part of molecular pathways determining the fate of neuronal progenitor cells, particularly in response to injury. ${ }^{22}$ Under oxidative conditions, indeed, SIRT1 activation suppressed proliferation of mouse cortical neural progenitor cells (NPCs) and directed their differentiation towards the astroglial lineage at the expense of the neuronal lineage. ${ }^{21}$ Thus, in such situations, SIRT1 may promote astroglial differentiation to facilitate astrogliosis and healing, which results in scarring at the damaged site. Through modulation of SIRT1, melatonin may regulate NPCs proliferation and differentiation. In cultured neural stem cells obtained from adult mouse subventricular zone, for example, melatonin promoted the differentiation of precursor cells into neuronal cells without affecting the number of glial cells. ${ }^{41}$ In addition, melatonin promoted proliferation of NPCs during hypoxia and induced their neuronal differentiation. ${ }^{42}$ We hypothesize that the differential expression of SIRT1 in neurons and glial cells found after melatonin treatment in the damaged brain may contribute to save neurons but may also 
direct the differentiation of NPCs toward a neuronal phenotype. In contrast with this hypothesis, however, it has been recently reported that in a model of white matter damage induced by hypoxia in the neonatal rat brain, SIRT1 activation regulated glial cell proliferation and promoted oligodendrocytes recovery. ${ }^{23}$ Further studies are therefore required to better define the role of SIRT1 and the effects of its modulators, including melatonin, in the regulation of the differentiation of NPCs after brain injury.

\section{5 | CONCLUSIONS}

We have demonstrated that SIRT1 is rapidly downregulated in neurons after HI both in the nucleus and in the cytoplasm. Administration of pharmacological doses of melatonin immediately after the ischemic insult rescued SIRT1 expression and activity, increased autophagy, and reduced necrotic and apoptotic cell death. We also show that glial cell activation was significantly reduced by melatonin and that reactive glial cells did not express SIRT1. These results add new insights into the protective effect of melatonin in this neonatal model of HI.

There is increasing evidence of a role of SIRT1 in NPCs differentiation. The different expression of SIRT1 in neurons and glial cells found after melatonin in the damaged side of the brain led us to hypothesize that, besides reducing cell death, melatonin might contribute to differentiate NPCs toward a neuronal phenotype. This effect could be particularly relevant for damaging events occurring during development because this is a period of intensive differentiation of NPCs. Of course, further experiments are required to sustain this hypothesis. 


\section{Acknowledgment}

Authors thank Claudia Scopa for the excellent technical assistance. This research was partially supported by grants from the University of Urbino Carlo Bo and the Europe Against Infant Brain Injury (EURAIBI) Foundation.

\section{Disclosure}

The authors declare they do not have any conflict of interest.

\section{Author Contributions}

S.C. conceptualized and designed the study, performed the acquisition of the data, drafted the initial manuscript, and approved the final manuscript as submitted. G.R. performed the acquisition of the data and approved the final manuscript as submitted. W.B. and G.B. designed the study, critically reviewed and revised the manuscript and approved the final manuscript as submitted. All authors approved the final manuscript as submitted and agree to be accountable for all aspects of the work. 


\section{REFERENCES}

1. Hendaus MA, Jomha FA, Alhammadi AH. Melatonin in the management of perinatal hypoxic-ischemic encephalopathy: light at the end of the tunnel? Neuropsychiatr Dis Treat. $2016 ; 12: 2473-2479$.

2. Sanchez-Barcelo EJ, Mediavilla MD, Reiter RJ. Clinical uses of melatonin in pediatrics. Int J Pediatr. 2011; 2011:892624.

3. Gitto E, Pellegrino S, Gitto P, et al. Oxidative stress of the newborn in the pre- and postnatal period and the clinical utility of melatonin. J Pineal Res. 2009; 46:128-139.

4. Reiter RJ, Mayo JC, Tan DX, et al. Melatonin as an antioxidant: under promises but over delivers. J Pineal Res. 2016; 61:253-278.

5. Ganie SA, Dar TA, Bhat AH, et al. Melatonin: A Potential Anti-Oxidant Therapeutic Agent for Mitochondrial Dysfunctions and Related Disorders. Rejuvenation Res. 2016; 19:21-40.

6. Korkmaz A, Reiter RJ. Epigenetic regulation: a new research area for melatonin? J Pineal Res. 2008; 44:41-44.

7. Jenwitheesuk A, Nopparat C, Mukda S, et al. Melatonin regulates aging and neurodegeneration through energy metabolism, epigenetics, autophagy and circadian rhythm pathways. Int J Mol Sci. 2014; 15:16848-16884.

8. Carrillo-Vico A, Lardone PJ, Alvarez-Sanchez N, et al. Melatonin: buffering the immune system. Int J Mol Sci. 2013; 14:8638-8683.

9. Carloni S, Albertini MC, Galluzzi L, et al. Melatonin reduces endoplasmic reticulum stress and preserves sirtuin 1 expression in neuronal cells of newborn rats after hypoxia-ischemia. $J$ Pineal Res. 2014; 57:192-199. 
10. Carloni S, Favrais G, Saliba E, Albertini MC, et al. Melatonin modulates neonatal brain inflammation through endoplasmic reticulum stress, autophagy, and miR-34a/silent information regulator 1 pathway. J Pineal Res. 2016; 61:370-380.

11. Brachmann CB, Sherman JM, Devine SE, et al. The SIR2 gene family, conserved from bacteria to humans, functions in silencing, cell cycle progression, and chromosome stability. Genes Dev. 1995; 9:2888-2902.

12. Tanno M, Sakamoto J, Miura T, et al. Nucleocytoplasmic shuttling of the NAD+-dependent histone deacetylase SIRT1. J Biol Chem. 2007; 282:6823-6832.

13. Stunkel W, Campbell RM. Sirtuin 1 (SIRT1): the misunderstood HDAC. J Biomol Screen. $2011 ; 16: 1153-1169$.

14. Martinez-Redondo P, Vaquero A. The diversity of histone versus nonhistone sirtuin substrates. Genes Cancer. 2013; 4:148-163.

15. Lan F, Cacicedo JM, Ruderman N, et al. SIRT1 modulation of the acetylation status, cytosolic localization, and activity of LKB1. Possible role in AMP-activated protein kinase activation. J Biol Chem. 2008; 283:27628-27635.

16. Ghosh HS, Mcburney M, Robbins PD. SIRT1 negatively regulates the mammalian target of rapamycin. PLoS One. 2010; 5:e9199.

17. Ng F, Tang BL. Sirtuins' modulation of autophagy. J Cell Physiol. 2013; 228:2262-2270.

18. Vaziri H, Dessain SK, Ng Eaton E, et al. hSIR2(SIRT1) functions as an NAD-dependent p53 deacetylase. Cell. 2001; 107:149-159.

19. Huang H, Tindall DJ. Dynamic FoxO transcription factors. J Cell Sci. 2007; 120:2479-2487.

20. Hallows WC, Lee S, Denu JM. Sirtuins deacetylate and activate mammalian acetyl-CoA synthetases. Proc Natl Acad Sci U S A 2006; 103:10230-10235. 
21. Prozorovski T, Schulze-Topphoff U, Glumm R, et al. Sirt1 contributes critically to the redoxdependent fate of neural progenitors. Nat Cell Biol. 2008; 10:385-394.

22. Libert S, Cohen D, Guarente L. Neurogenesis directed by Sirt1. Nat Cell Biol. 2008; 10:373374.

23. Jablonska B, Gierdalski M, Chew LJ, et al. Sirt1 regulates glial progenitor proliferation and regeneration in white matter after neonatal brain injury. Nat Commun. 2016; 7:13866.

24. Dali-Youcef N, Lagouge M, Froelich S, et al. Sirtuins: the 'magnificent seven', function, metabolism and longevity. Ann Med. 2007; 39:335-345.

25. Carloni S, Carnevali A, Cimino M, et al. Extended role of necrotic cell death after hypoxiaischemia-induced neurodegeneration in the neonatal rat. Neurobiol Dis. 2007; 27:354-361.

26. Nijboer $\mathrm{CH}$, Groenendaal F, Kavelaars A, et al. Gender-specific neuroprotection by 2iminobiotin after hypoxia-ischemia in the neonatal rat via a nitric oxide independent pathway. J Cereb Blood Flow Metab. 2007; 27:282-292.

27. Carloni S, Girelli S, Scopa C, et al. Activation of autophagy and Akt/CREB signaling play an equivalent role in the neuroprotective effect of rapamycin in neonatal hypoxia-ischemia. Autophagy. 2010; 6:366-377.

28. Mouchiroud L, Houtkooper RH, Moullan N, et al. The NAD(+)/Sirtuin Pathway Modulates Longevity through Activation of Mitochondrial UPR and FOXO Signaling. Cell. 2013; $154: 430-441$.

29. Zhao J, Brault JJ, Schild A, Cao et al. FoxO3 coordinately activates protein degradation by the autophagic/lysosomal and proteasomal pathways in atrophying muscle cells. Cell Metab. $2007 ; 6: 472-483$.

30. Lee IH, Cao L, Mostoslavsky R, et al. A role for the NAD-dependent deacetylase Sirt1 in the regulation of autophagy. Proc Natl Acad Sci U S A 2008; 105:3374-3379. 
31. Zhao S, Xu W, Jiang W, et al. Regulation of cellular metabolism by protein lysine acetylation. Science. 2010; 327:1000-1004.

32. Chang HC, Guarente L. SIRT1 and other sirtuins in metabolism. Trends Endocrinol Metab. $2014 ; 25: 138-145$.

33. Rodriguez RM, Fernandez AF, Fraga MF. Role of sirtuins in stem cell differentiation. Genes Cancer. 2013; 4:105-111.

34. Hernandez-Jimenez M, Hurtado O, Cuartero MI, et al. Silent information regulator 1 protects the brain against cerebral ischemic damage. Stroke. 2013; 44:2333-2337.

35. Lee JT, Gu W. SIRT1: Regulator of p53 Deacetylation. Genes Cancer. 2013; 4:112-117.

36. Hariharan N, Maejima Y, Nakae J, et al. Deacetylation of FoxO by Sirt1 Plays an Essential Role in Mediating Starvation-Induced Autophagy in Cardiac Myocytes. Circ Res. 2010; 107:1470-1482.

37. Sheng R, Zhang LS, Han R, et al. Autophagy activation is associated with neuroprotection in a rat model of focal cerebral ischemic preconditioning. Autophagy. 2010; 6:482-494.

38. Jiang T, Yu JT, Zhu XC, et al. Acute metformin preconditioning confers neuroprotection against focal cerebral ischaemia by pre-activation of AMPK-dependent autophagy. $\mathrm{Br} J$ Pharmacol. 2014; 171:3146-3157.

39. Salminen A, Kaarniranta K. SIRT1: regulation of longevity via autophagy. Cell Signal. 2009; 21:1356-1360.

40. Wu Y, Li X, Zhu JX, et al. Resveratrol-activated AMPK/SIRT1/autophagy in cellular models of Parkinson's disease. Neurosignals. 2011; 19:163-174.

41. Sotthibundhu A, Phansuwan-Pujito P, Govitrapong P. Melatonin increases proliferation of cultured neural stem cells obtained from adult mouse subventricular zone. J Pineal Res. 2010; 49:291-300. 
42. $\mathrm{Fu}$ J, Zhao SD, Liu HJ, et al. Melatonin promotes proliferation and differentiation of neural stem cells subjected to hypoxia in vitro. J Pineal Res. 2011; 51:104-112. 


\section{FIGURE LEGENDS}

FIGURE 1 Effect of neonatal hypoxia-ischemia and melatonin on propidium iodide (PI) uptake.

A) Representative photomicrographs showing cells labeled with PI (red) in the lesioned cerebral cortex and lesioned CA1 region of the hippocampus of vehicle-treated (HI) and melatonin-treated (HI+Mel) ischemic animals. PI was injected icv $20 \mathrm{~min}$ before the sacrifice of the animals. Scale bar, $100 \mu \mathrm{m}$. B) PI-positive cells counting in the cerebral cortex, CA1 and CA2/CA3 regions of the hippocampus. Counting was performed as described in Methods and data reported as the mean \pm S.E.M. (N=5/group). ${ }^{*} \mathrm{p}<0.05, \S \mathrm{p}<0.001$, One-way ANOVA followed by Newman-Keuls Multiple Comparison Test.

FIGURE 2 Effect of neonatal hypoxia-ischemia and melatonin on mitochondrial apoptosis.

Representative Western blots and quantitative evaluation of $\mathrm{Bax}(\mathrm{A}, \mathrm{B})$ and cytochrome c $(\mathrm{C}, \mathrm{D})$ expression in the mitochondrial and the cytosolic fraction of the cerebral cortex of vehicle-treated (C) or melatonin-treated $(\mathrm{C}+\mathrm{Mel})$ control animals and vehicle-treated $(\mathrm{HI})$ or melatonin-treated $(\mathrm{HI}+\mathrm{Mel})$ ischemic animals sacrificed $1 \mathrm{~h}$ after HI. $\beta$-actin or Hsp60 were run as loading controls for the cytosolic and the mitochondrial fraction, respectively. Data are expressed as \% of control and are the mean \pm S.E.M. ( $\mathrm{N}=8$ /group). E) Representative Western blot and quantitative evaluation of cleaved caspase- 3 expression in the cerebral cortex of vehicle-treated $(\mathrm{C})$ or melatonin-treated $(\mathrm{C}+\mathrm{Mel})$ control animals of vehicle-treated (HI) or melatonin-treated (HI+Mel) ischemic animals sacrificed $6 \mathrm{~h}$ after HI. $\beta$-actin was run as loading control. Data are expressed as OD integration and are the mean \pm S.E.M. (N=8/group). L, left side, contralateral; R, right side, ipsilateral to the occluded carotid artery; nd, non detectable. ${ }^{*} \mathrm{p}<0.05, * * \mathrm{p}<0.01$ vs. control group, One-way ANOVA followed by Newman-Keuls Multiple Comparison Test. 
FIGURE 3 Effect of neonatal hypoxia-ischemia and melatonin on SIRT1 expression.

Photomicrographs (A) of experiments performed $1 \mathrm{~h}$ after HI showing cells expressing SIRT1 (green) in the lesioned (R) cerebral cortex and the CA1 area of the hippocampus of vehicle-treated (HI) or melatonin-treated $(\mathrm{HI}+\mathrm{Mel})$ ischemic animals. $\mathrm{N}=5 /$ group. Scale bars, $100 \mu \mathrm{m}$. Representative Western blot and quantitative evaluation of SIRT1 expression in the whole cerebral cortex (B) and in the cytosolic (C) and the nuclear (D) fraction of the cerebral cortex of vehicle-treated (C) or melatonin-treated $(\mathrm{C}+\mathrm{Mel})$ control animals and vehicle-treated $(\mathrm{HI})$ or melatonin-treated $(\mathrm{HI}+\mathrm{Mel})$ ischemic animals sacrificed $1 \mathrm{~h}$ after HI. $\beta$-actin or Lamin B (LMN B) were run as loading controls for the cytosolic and the nuclear fraction, respectively. Data are expressed as $\%$ of control or as OD integration, and are the mean \pm S.E.M. ( $N=8$ /group). L, left side, contralateral; $\mathrm{R}$, right side, ipsilateral to the occluded carotid artery. ${ }^{* *} \mathrm{p}<0.01, \S \mathrm{p}<0.001$ vs. control group, One-way ANOVA followed by Newman-Keuls Multiple Comparison Test.

FIGURE 4 Effect of neonatal hypoxia-ischemia and melatonin on p53 expression.

Representative Western blots and quantitative evaluation of p53 (A, B) and acetyl-p53 (C, D) expression in the cytosolic and the nuclear fraction of the cerebral cortex of vehicle-treated (C) or melatonin-treated $(\mathrm{C}+\mathrm{Mel})$ control animals and vehicle-treated $(\mathrm{HI})$ or melatonin-treated $(\mathrm{HI}+\mathrm{Mel})$ ischemic animals sacrificed 1h after HI. $\beta$-actin or Lamin B (LMN B) were run as loading controls for the cytosolic and the nuclear fraction, respectively. Data are expressed as $\%$ of control, and are the mean \pm S.E.M. ( $N=8$ /group). L, left side, contralateral; $\mathrm{R}$, right side, ipsilateral to the occluded carotid artery. ${ }^{* *} \mathrm{p}<0.01, \S \mathrm{p}<0.001$ vs. control group, One-way ANOVA followed by NewmanKeuls Multiple Comparison Test.

FIGURE 5 Effect of neonatal hypoxia-ischemia and melatonin on autophagy markers. 
Representative Western blots and quantitative evaluation of lipidated LC3 (LC3 II, A) and p62 (B) expression in the cerebral cortex of vehicle-treated $(\mathrm{C})$ or melatonin-treated $(\mathrm{C}+\mathrm{Mel})$ control animals and vehicle-treated $(\mathrm{HI})$ or melatonin-treated $(\mathrm{HI}+\mathrm{Mel})$ ischemic animals sacrificed $1 \mathrm{~h}$ after HI. $\beta$ actin was run as loading control. Data are expressed as $\%$ of control, and are the mean \pm S.E.M. ( $\mathrm{N}=8$ /group). L, left side, contralateral; $\mathrm{R}$, right side, ipsilateral to the occluded carotid artery. ** $\mathrm{p}<0.01, \S \mathrm{p}<0.001$ vs. control group, One-way ANOVA followed by Newman-Keuls Multiple Comparison Test.

FIGURE 6 Effect of neonatal hypoxia-ischemia and melatonin on glial fibrillary acidic protein (GFAP) expression.

A) Representative photomicrographs of GFAP-positive cells in the lesioned (R) cerebral cortex of vehicle-treated $(\mathrm{HI})$ and melatonin-treated $(\mathrm{HI}+\mathrm{Mel})$ ischemic animals. The lower right part of the figure shows the cortical area analyzed in the immunohistochemical experiments reported in the left part of the figure. Scale bar, $50 \mu \mathrm{m}$. B) Quantitative evaluation of GFAP expression in the cerebral cortex of vehicle-treated control animals (C) and vehicle-treated (HI) or melatonin-treated (HI+Mel) ischemic animals sacrificed $1 \mathrm{~h}$ after HI. Data are expressed as $\%$ of control, and are the mean \pm S.E.M. ( $\mathrm{N}=8$ /group). ${ }^{*} \mathrm{p}<0.05, \S \mathrm{p}<0.001$ vs. control group, One-way ANOVA followed by Newman-Keuls Multiple Comparison Test.

FIGURE 7 SIRT1 expression in neuronal and glial cells.

Photomicrographs of experiments performed $1 \mathrm{~h}$ after HI showing cells labeled in the parietal cerebral cortex of vehicle-treated control animals (Control) and in the lesioned (R) cerebral cortex of vehicletreated (HI) or melatonin-treated (HI+Mel) ischemic animals with: (A) SIRT1 (panels a, d, g; green) and GFAP (panels b, e and h; red); (B) SIRT1 (panels 1, o and r; green) and NeuN (panels m, p and 
s; red). Merged images (c, f, i, n, q, t) illustrate co-localization of SIRT1 with GFAP (c, f, i; arrows, yellow) or with NeuN (n, q, t; yellow). N=5/group. Scale bars, $100 \mu \mathrm{m}$. 
(A)

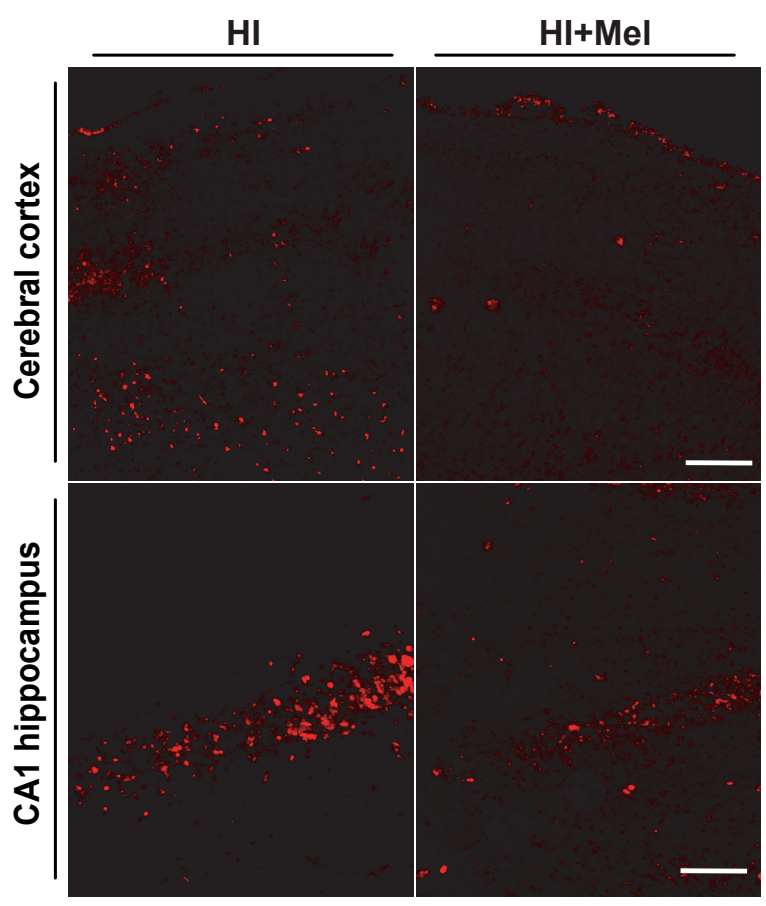

(B) 150

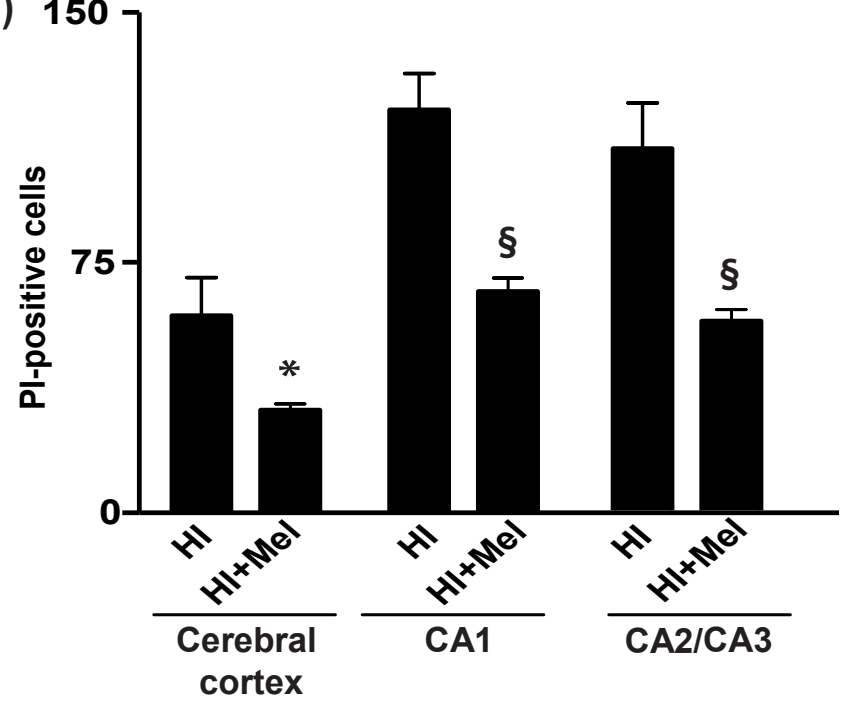



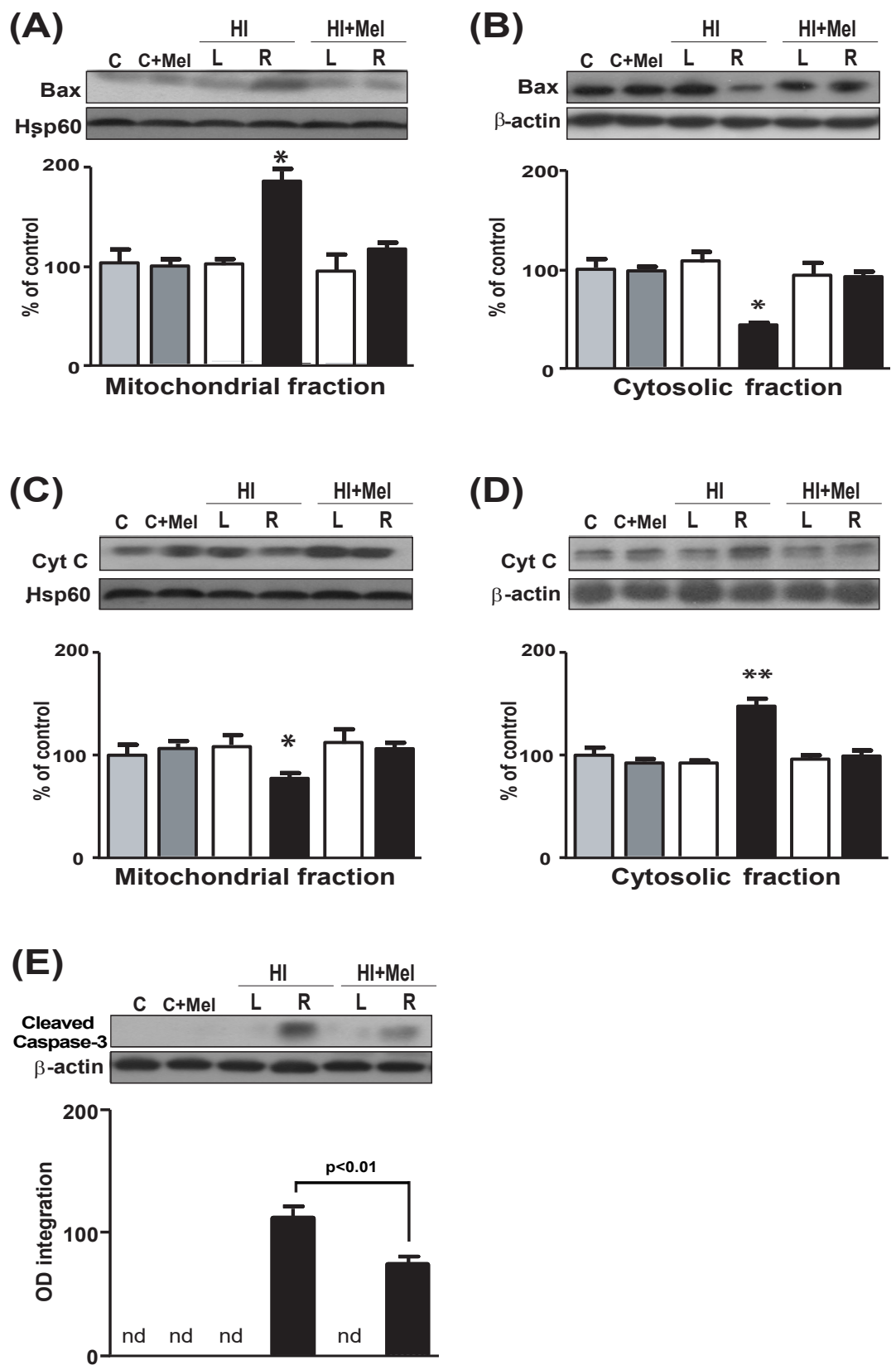
(A)

$$
\text { HI,R }
$$

HI+Mel,R

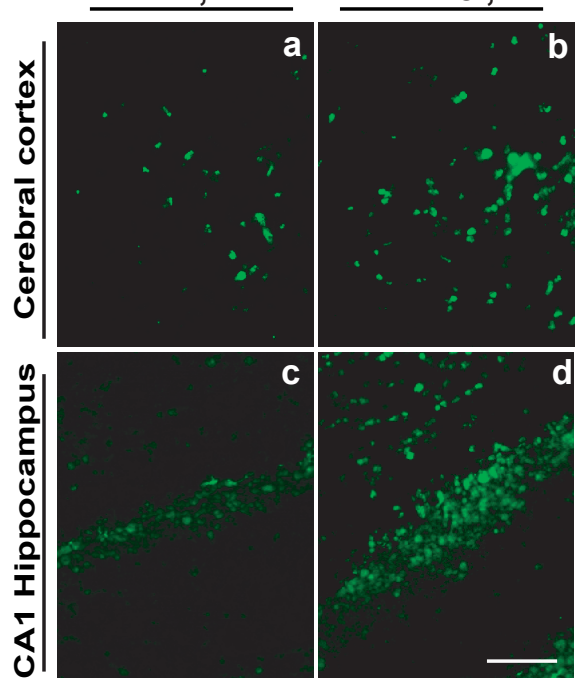

(C)

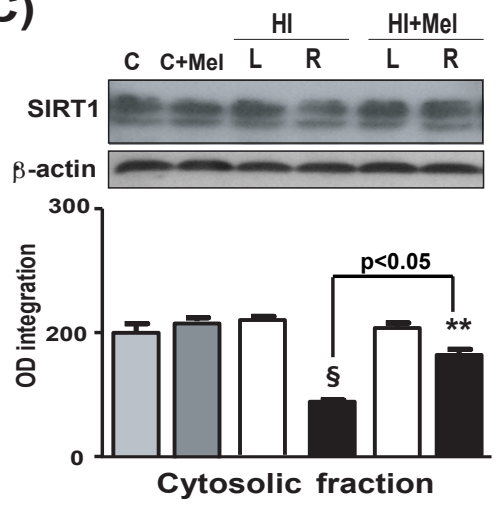

(B)

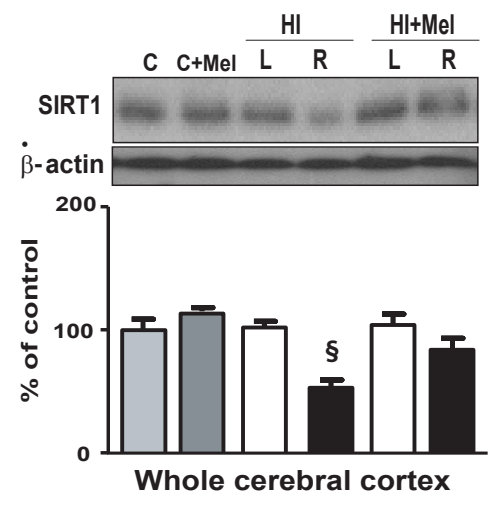

(D)

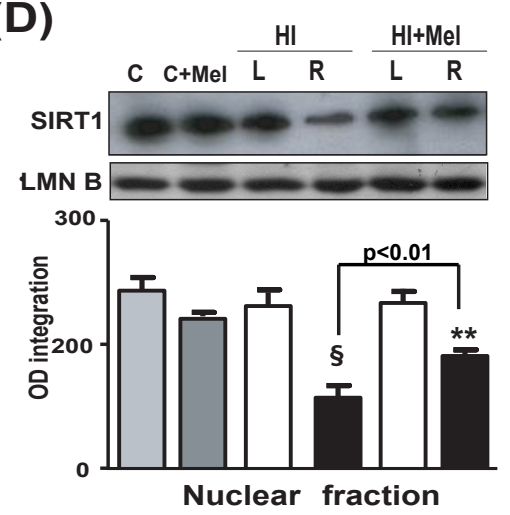


(A)

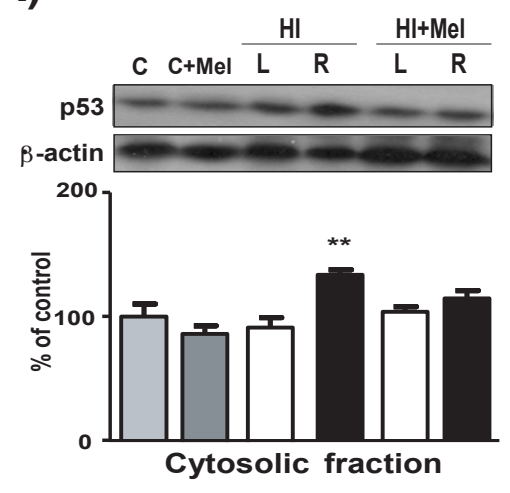

(C)

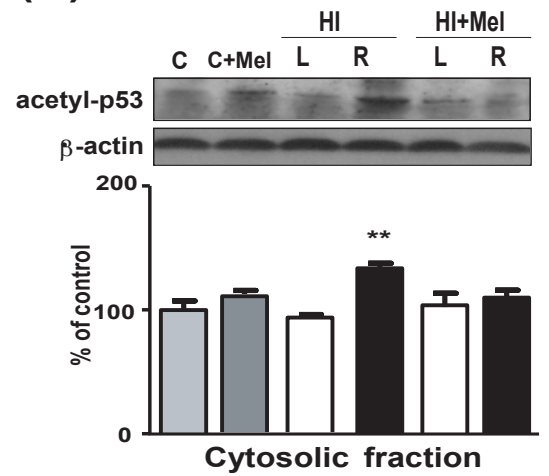

(B)

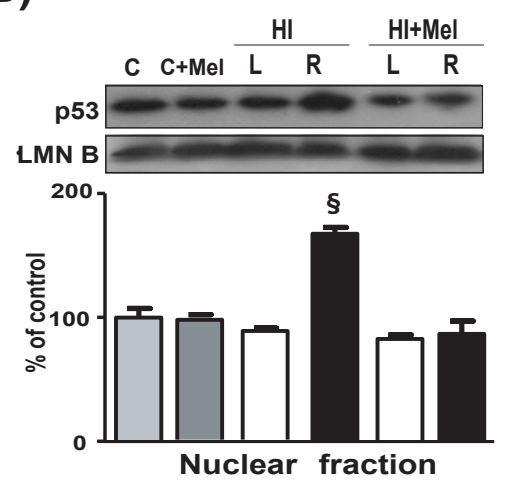

(D)

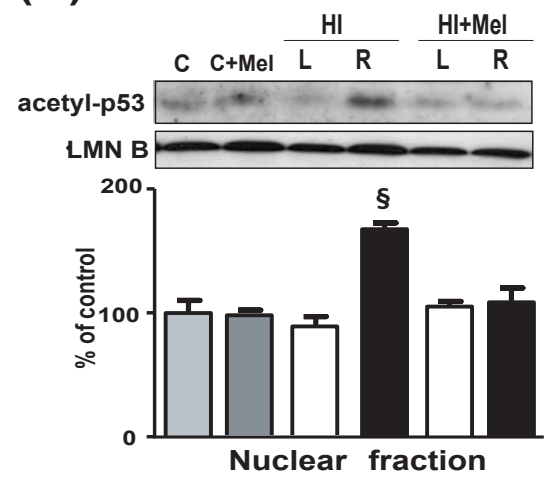




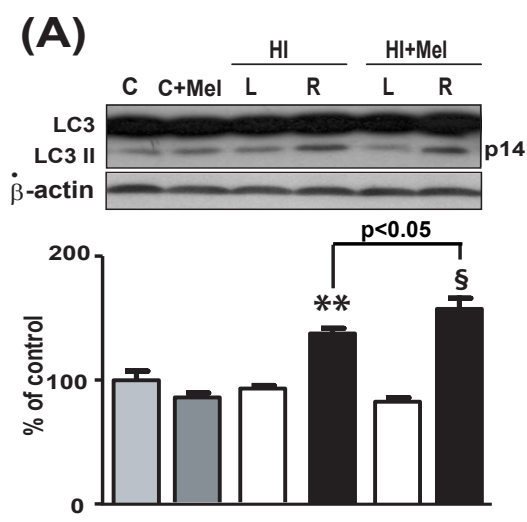

(B)

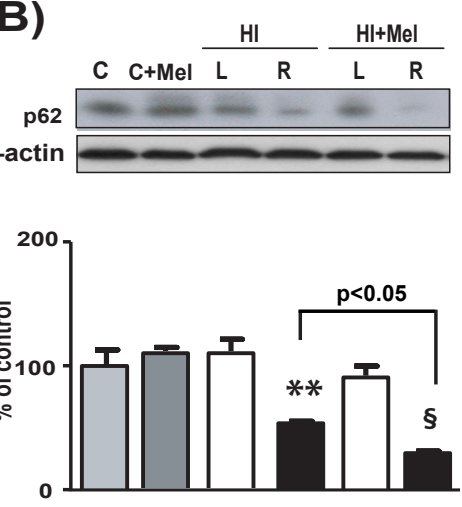




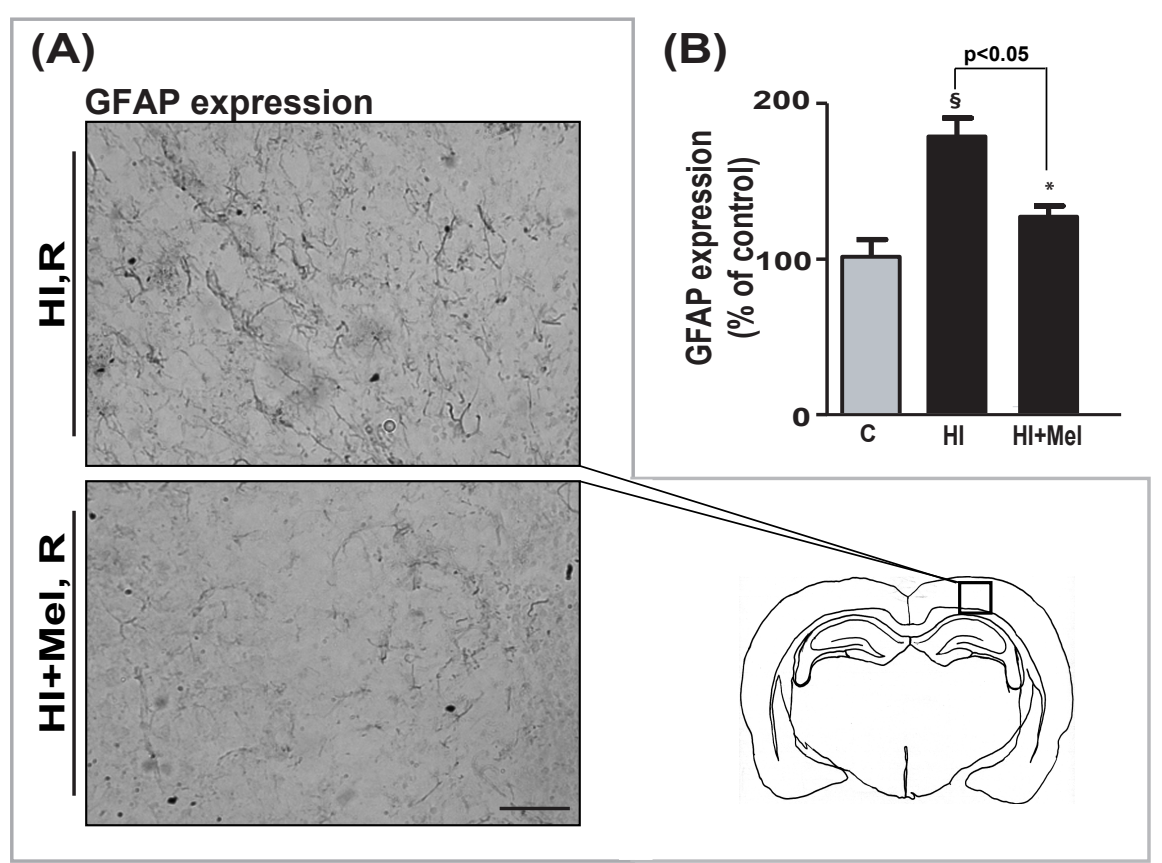



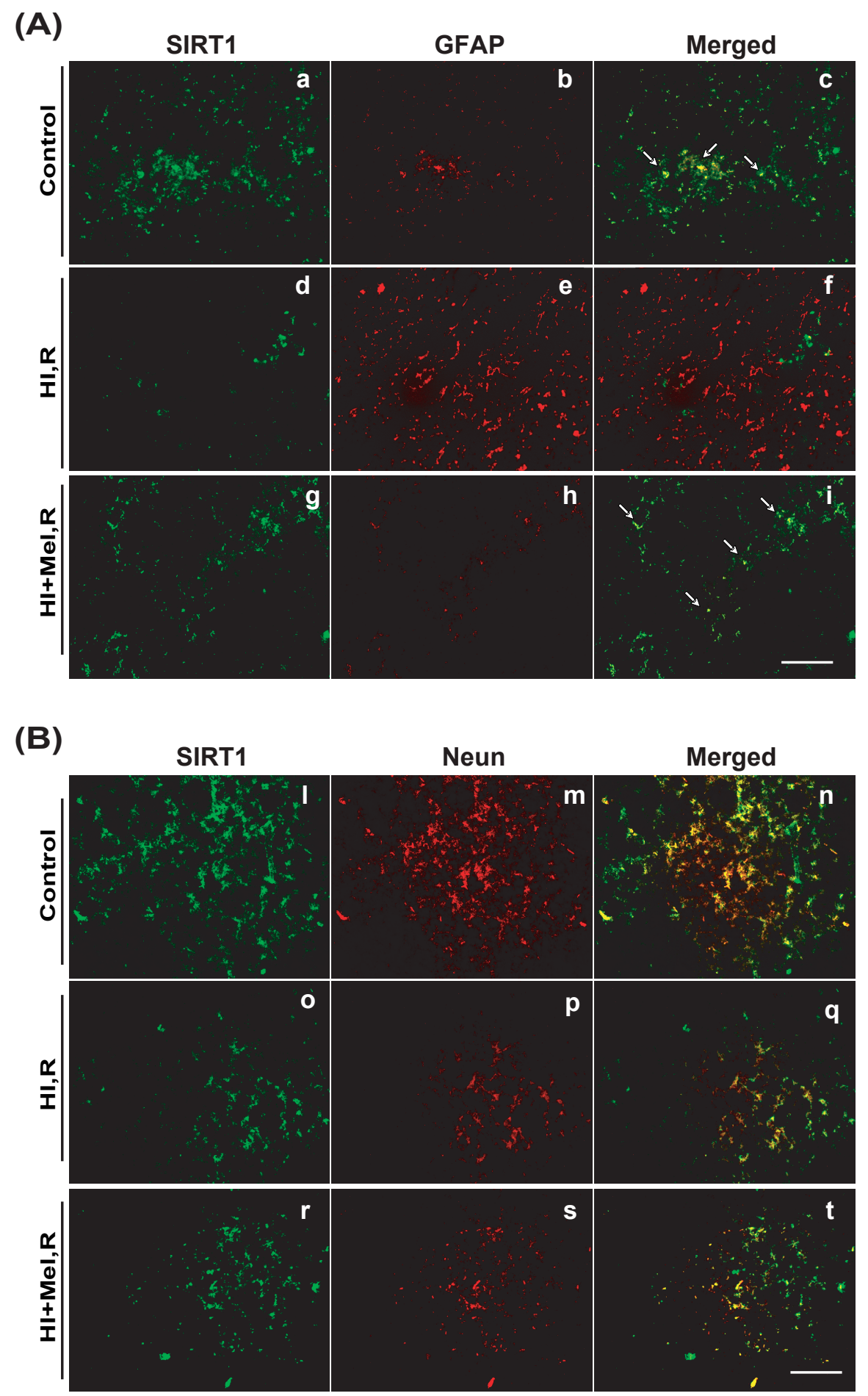